\title{
Novel ACTA1 mutation causes late-presenting nemaline myopathy with unusual dark cores
}

\author{
Matteo Garibaldi ${ }^{a, *}$, Fabiana Fattori ${ }^{b}$, Elena Maria Pennisi ${ }^{c}$, Gioia Merlonghi ${ }^{a}$, Laura Fionda ${ }^{a}$, \\ Fiammetta Vanoli ${ }^{a}$, Luca Leonardi ${ }^{a}$, Elisabetta Bucci ${ }^{a}$, Stefania Morino ${ }^{a}$, Andrea Micaloni ${ }^{d}$, \\ Tommaso Tartaglione ${ }^{\mathrm{e}}$, Bas Uijterwijk ${ }^{\mathrm{f}}$, Martijn Zierikzee ${ }^{\mathrm{f}}$, Coen Ottenheijm ${ }^{\mathrm{f}}$, \\ Enrico Silvio Bertini ${ }^{b}$, Antonella Stoppacciaros, Salvatore Raffa ${ }^{\mathrm{d}}$, Marco Salvetti ${ }^{\mathrm{a}}$, \\ Giovanni Antonini ${ }^{\text {a }}$ \\ ${ }^{a}$ Neuromuscular and Rare Disease Centre, Department of Neuroscience, Mental Health and Sensory Organs (NESMOS), SAPIENZA University of Rome, \\ Sant'Andrea Hospital, Rome, Italy \\ ${ }^{\mathrm{b}}$ Unit of Neuromuscular and Neurodegenerative Disorders, Laboratory of Molecular Medicine, Bambino Gesù Children's Research Hospital, Rome, Italy \\ ${ }^{\mathrm{c}}$ Unit of Neuromuscular Disorders, Neurology, San Filippo Neri Hospital, Rome, Italy \\ ${ }^{\mathrm{d}}$ Laboratory of Ultrastructural pathology, Department of Clinical and Molecular Medicine, SAPIENZA University of Rome, Sant'Andrea Hospital, Rome, Italy \\ ${ }^{\mathrm{e}}$ Department of Radiology, Istituto Dermopatico dell'Immacolata, IRCCS, Rome, Italy \\ ${ }^{\mathrm{f}}$ Department of Physiology, Amsterdam UMC (location VUmc), Amsterdam, Netherlands \\ $\mathrm{g}^{\mathrm{g}}$ Unit of Pathology, Department of Clinical and Molecular Medicine, SAPIENZA University of Rome, Sant'Andrea Hospital, Rome, Italy
}

Received 24 May 2020; received in revised form 19 November 2020; accepted 25 November 2020

Available online xxx

\begin{abstract}
ACTA1 gene encodes the skeletal muscle alpha-actin, the core of thin filaments of the sarcomere. ACTAl mutations are responsible of several muscle disorders including nemaline, cores, actin aggregate myopathies and fiber-type disproportion. We report clinical, muscle imaging, histopatological and genetic data of an Italian family carrying a novel ACTA1 mutation. All affected members showed a latepresenting, diffuse muscle weakness with sternocleidomastoideus and temporalis atrophy. Mild dysmorphic features were also detected. The most affected muscles by muscle MRI were rectus abdominis, gluteus minimus, vastus intermedius and both gastrocnemii. Muscle biopsy showed the presence of nemaline bodies with several unusual dark areas at Gomori Trichrome, corresponding to unstructured cores with abundant electrodense material by electron microscopy. The molecular analysis revealed missense variant $\mathrm{c} .148 \mathrm{G}>A$; $\mathrm{p} .(\mathrm{Gly} 50 \mathrm{Ser})$ in the exon 3 of ACTA1, segregating with affected members in the family. We performed a functional essay of fibre contractility showing a higher $\mathrm{pCa}_{50}$ (a measure of the calcium sensitivity of force) of type 1 fibers compared to control subjects' type 1 muscle fibers. Our findings expand the clinico-pathological spectrum of ACTAl-related congenital myopathies and the genetic spectrum of core-rod myopathies.

(C) 2020 Elsevier B.V. All rights reserved.
\end{abstract}

Key Words: Acta1; Nemaline myopathy; Central core; Congenital myopathy; Core-rod myopathy; Central core disease.

\section{Introduction}

Actinopathies represent a specific subgroup of congenital myopathies with protein accumulation in muscle biopsy due to mutations in the skeletal muscle $\alpha$-actin gene

\footnotetext{
* Corresponding author.

E-mail address: matteo.garibaldi@uniroma1.it (M. Garibaldi).
}

(ACTA1) [1,2]. Both dominant and recessive traits have been reported, resulting in variable protein expression [3-9] and dysfunctional sarcomere contractility $[10,11]$. The observation of sporadic patients with de novo dominant mutations suggests a high new mutation rate in ACTAl [12,13]. The most common morphological findings are nemaline bodies - rod-like structures - which typically accumulates in subsarcolemmal areas $[2,8,14,15]$. Intranuclear rods have also 
been described [4,16-18]. Besides nemaline bodies, several histopathological findings have been reported in association to ACTA1 mutations including actin filament aggregates [9,19], cores [20], caps [21], fiber type disproportion [22-24] and zebra bodies [25]. ACTA1-related nemaline myopathies account for about $20 \%$ of all nemaline myopathies and $50 \%$ of the severe cases, representing the most common clinical presentation $[6,9,14,26]$. Clinical picture is characterized by marked hypotonia at birth, myopathic face, high arched palate, respiratory failure and feeding difficulties, with death occurring within the first year of life or severe muscular weakness requiring mechanical ventilation in those who survive $[13,17,27,28]$. However, a wide range of clinical presentations has been reported, ranging from fetal akinesia syndrome [29] to milder phenotypes with adult onset [8,30,31]. Infrequently, ACTA1-myopathies can manifest with atypical clinical and histopathological findings, as facioscapuloperoneal myopathy [30], congenital muscular dystrophy with rigid spine [7], muscular stiffness and hypertonia [32], distal weakness with rimmed vacuoles [33], myofibrillar aggregates [28] or cytoplasmic bodies without nemaline bodies [34].

Herein we describe an Italian family manifesting a latepresenting core-rod myopathy with peculiar morphological elements at muscle biopsy due to a novel ACTA1 mutation.

\section{Materials and methods}

\subsection{Patients}

All patients underwent a complete clinical examination including extensive manual muscle test scored by Medical Research Council (MRC) and laboratory analysis including creatine kinase $(\mathrm{CK})$. Proband and her sister (the most affected patients) also underwent a neurophysiological study including nerve conduction study (NCS) and electromyography (EMG), whole body muscle MRI including T1 and STIR sequences, open muscle biopsy and targetedNGS panel for congenital myopathies. In order to confirm the correct segregation of novel ACTA1 mutation in the family we searched for ACTAl mutation by Sanger analysis in all affected members.

\subsection{Morphological study}

Open muscle biopsies were obtained from two patients: proband (PII.4) and her older sister (PII.1) at age of 39 (vastus lateralis and biceps brachii) and 55 years (vastus lateralis) respectively, basing on clinical and muscle MRI findings. For conventional histological and histochemical techniques we used the already reported protocol [35], as well as Congo Red stain and immunohistochemical (IHC) study to better characterize the atypical cores, including myosins fast (WBMHCf, Monosan), slow (WB-MHCs, Monosan), neonatal (WB-MHCn, Monosan) and developmental (RNMy2/9D2,
Monosan), myotilin (polyclonal, GeneTex), desmin (D33, Dako), alpha-B crystallin (CRYAB Polyclonal Antibody, ThermoFisher Scientific) revealed by peroxidase. Digital photographs of biopsies were obtained with a Nikon Eclipse E-200 microscope linked to a Nikon Digital Sight DS-Fi1 (Nikon Corporation, Japan).

\subsection{Muscle MRI}

Lower limbs and scapular girdle muscle imaging was obtained by a 1.5T MRI device, following the already reported internal protocol in accordance to the international consensus recommendations [36,37]. Fibro-fatty replacement was evaluated in T1-sequences accordingly to Mercuri scale modified by Fisher [38], whereas muscular oedemainflammation was evaluated in STIR images as positive hyperintense signal.

\subsection{Molecular analysis}

Genomic DNA was extracted from peripheral blood with standard methods after receiving informed consent.

Next Generation Sequencing analysis was performed using target enrichment method on Illumina platform with a uniquely customized panel for congenital myopathy including 95 genes (NEB, MYO18B, ACTA1, TPM2, TPM3, KBTBD13, KLHL40, KLHL41, LMOD3, TNNT1, CFL2, MYPN, DNM2, MTM1, SPEG1, CCDC78, BIN1, RYR1, MYH2, MYH7, SEPN1, STIM1, ORAI1, PGAM2, MEGF10, MTMR14, TRIM32, FHL1, HACD1, C-term TTN, DNA2, STAC3, CACNA1S, ZAK, VMA21, SCN4A, CLCN1, VCP, GNE, KLHL9, HSPB8, ADSSL1, SQSTM1, MATR3, TIA1, BAG3, CRYAB, DES, FLNC, PYROXD1, KY, LDB3, HNRPDL, LMNA, FKRP, DYSF, CAPN3, FKTN, TORIAIP1, GTDC2, GMPPB, LAMA2, COL6A1, COL6A2, COL6A3, COL12A1, ANO5, PYGM, CPT2, CAV3, DMD, EMD, CHKB, INPP5K, PLEC, POMT1, POMT2, POMGNT1, DPM1, DPM2, DPM3 FCMD, LARGE, B3GALNT2, B4GAT1, COLAA1, DAG1, ISPD, POMK, TMEM5, SGCG, SGCA, SGCB, SGCD, SYNE1, SYNE2, TMEM43, PTRH2, ITGA7, TRAPPC11, DNAJB6, MYOT, TNPO3, TCAP, RAPSN, MUSK, DOK7, LAMB2, COLQ, GFPT, CHRNE, DPAGT1, CHAT, AGRN, CHRNA1, CHRNB1, CHRND, SMN1, ASCC1, TRIP4.). DNA sample was enriched using custom probes with the Nextera Rapid Capture Custom Enrichment Kit (Illumina, San Diego, California, USA) following the manufactures instructions. Specific regions of interest were captured by hybridization to biotinylated probes and then isolated by magnetic pulldown. DNA capture, enrichment and paired-end sequencing with read length of $151 \mathrm{bp}$ were performed using Illumina MiSeq with a sequencing depth of $100 \mathrm{X}$. The Illumina VariantStudio data analysis software was used to annotate the variants. Sanger sequencing of exon 3 of ACTA1 (NM_001100.3) was performed to confirm the novel variant identified by NGS in proband and in her relatives. 


\subsection{Functional assay of fiber contractility}

We adapted previously described methods to investigate the contractile properties of myofibers from the PII.1 muscle biopsy and from biopsies of healthy controls [39]. Small sections $(2 \times 2 \mathrm{~mm})$ were isolated from the biopsies and glycerinated for $24 \mathrm{~h}$ at $-20^{\circ} \mathrm{C}$. Single myofibers from control subjects (average age at biopsy: $47 \pm 8$ years) and the patient were dissected from the muscle strips and clipped between aluminium foil T-clips. Myofibers were permeabilized in $1 \%(\mathrm{v} / \mathrm{v})$ Triton $^{\mathrm{TM}} \mathrm{X} 100 /$ relaxing solution, then mounted between a length motor (ASI 315C I; Aurora Scientific, Aurora, ON, Canada) and a force transducer element (ASI 403A, Aurora Scientific) in a permeabilized myofiber apparatus (ASI 802D, Aurora Scientific) mounted on top of an inverted microscope (Axio Observer A1; Zeiss, Oberkochen, Germany). Sarcomere length was set to $2.5 \mu \mathrm{m}$ using a 40x objective, high-speed VSL camera and ASI 900B software (Aurora Scientific). Myofiber length, width, and depth were measured at three points along the myofiber using a 10x objective, a prism, and a custom-made mirror mounted in the bath. Cross-sectional area was calculated with the average width and depth of the myofiber assuming an elliptical cross-section. To determine the $\mathrm{Ca}^{2+}$-sensitivity of force generation and Hill slope, permeabilized single myofibers were exposed to solutions with incremental $\mathrm{Ca}^{2+}$ concentration increases. Steady-state forces were measured at each $\mathrm{Ca}^{2+}$-concentration, then these steady-state values were normalized to the maximal force obtained at $\mathrm{pCa} 4.5$. The force-pCa data were fitted to the Hill equation $(Y=1 /$ $\left.\left[1+10 \mathrm{n}_{\mathrm{H}}\left(\mathrm{pCa}-\mathrm{pCa}_{50}\right)\right]\right)[40,41]$. The criteria of acceptance regarding the myofiber contractility studies included: (1) preserved structural integrity of the myofiber as indicated by the striation pattern (also required to set sarcomere length); (2) the force at the final pCa 4.5 had to be higher than $90 \%$ of the force during pre-activation. Each myofiber underwent two maximal activations ( $\mathrm{pCa} 4.5$ ) and five submaximal activations (pCa 7.0 - 6.0 - 5.8 - 5.6 - 5.4); (3) preserved sarcomere length in the myofibers after completion of the experimental protocol (to assure that the myofiber was well set in the clip). Applying these criteria, $~ 80 \%$ of the myofiber experiments were included in the results shown. Determination of myosin heavy chain composition of measured myofibers was performed as described previously $[42,43]$.

\section{Results}

\subsection{Clinical findings}

Proband (PII.4) is a 53-year-old woman, with a negative medical history, referred to our neuromuscular centre at the age of 39 just because of occasional Creatin Kinase (CK) elevation (range 90-1500 U/l). Since her infancy, she was having some difficulties in physical activities and presented mild rhinolalia, which had never been considered pathological by the patient. Clinical examination at age of 39 revealed a mild myopathic face with high arched palate, atrophy of sternocleidomastoideus (SCM) and temporalis muscles, weakness of neck flexors and mild diffuse muscle weakness, never noticed before by the patient. Clinical evolution over 14 years of follow-up consisted in a very slow progression of muscle weakness. Electromyography (EMG) revealed myopathic changes and occasional pseudomyotonic discharges.

Her 57-years-old sister (PII.1) presented similar but more severe clinical phenotype consisting in mild myopathic face, SCM atrophy and diffuse, proximal-predominant, muscular weakness since her fifties (Fig. 1). Her 33 years-old son (PIII.2), referred as asymptomatic, had myopathic face with high arched palate, SCM atrophy with neck flexor weakness, and mild distal weakness of upper limbs. Similarly, the son of the proband (PIII.4; 31 years-old) presented a myopathic face with high arched palate and SCM atrophy with neck flexor weakness, without limb muscular weakness. The 72-years-old father of the proband (PI.1) and the old aunt (p.I.2; 64 years old) presented similar findings with late-manifesting diffuse muscular weakness (Fig. 2). All proband's relatives had normal CK level. Respiratory and cardiac evaluations were unremarkable in all affected subjects.

\subsection{Muscle imaging data}

Muscle MRI of lower limbs was obtained from patient II.1 and II.4. The most affected muscles in both studies weregluteus minimus, rectus abdominis, gastrocnemii, and vastus intermedius (Fig. 3). This pattern was more evident in the older sister (PII.1) who also showed a prominent involvement of the other vasti, whereas in the proband (PII.4) only the distal part of vastus intermedius was affected. Posterior muscles of the thighs were also mildly affected, particularly biceps femoris (long head). In lower legs, both medial and lateral gartrocnemi were selectively involved and represented the most affected muscles in both patients respectively.

Scapular girdle study, available for PII.1, showed complete atrophy of SCM, and mild to moderate fibro-fatty replacement of paravertebralis, latissimus dorsi and serratus anterior muscles (Fig. 3).

The overall muscle involvement was symmetric and STIR sequences did not show any positive signal in both patients.

\subsection{Morphological features}

Muscle biopsy in PII.4, performed in biceps brachii and vastus lateralis at 39 years, revealed myopathic changes with fibre size variability and prominent nuclear internalization, without fiber type predominance (Fig. 4,A). Scattered cytoplasmic and subsarcolemmal nemaline bodies were detected in some fibres (Fig. 4,B). Several muscle fibres showed cytoplasmic areas of dark material deposition with 


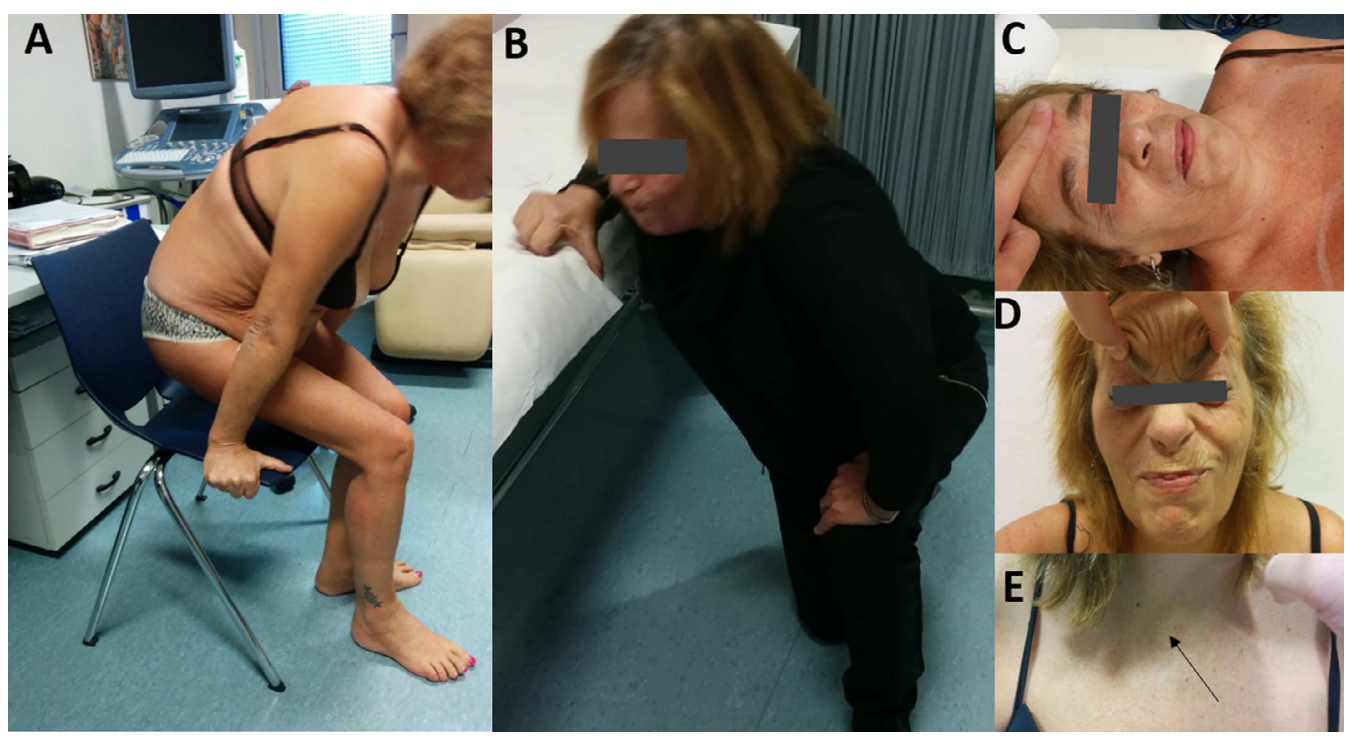

Fig. 1. Clinical picture.

Clinical manifestations in PII.1: mild proximal weakness (A,B) neck flexors (C) and orbicularis oculi (D) weakness with sternocleidomastoideus atrophy (E, arrow).

I.

II.

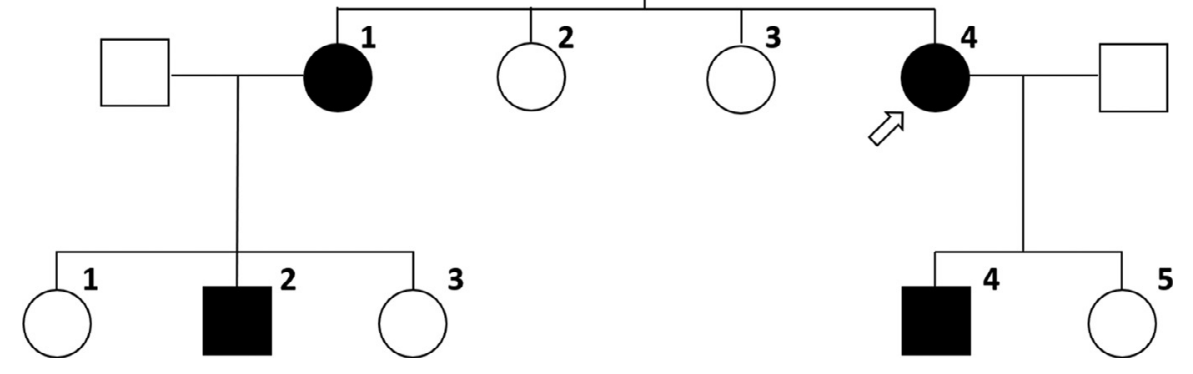

Fig. 2. Family pedigree.

All affected family members in black: the proband (PII.4, arrow), her older sister(PII.1), her father (PI.1), her aunt (PI.2), her son (PIII.4) and nephew (PIII.2).

perilesional halo at Gömöri trichrome (GT) stain (Fig. 4,C,D), corresponding to irregular areas lacking enzymatic activity at oxidative stains (Fig. 4,E). Similar histopathological findings were detected in the muscle biopsy of the older sister II.1 collected from vastus lateralis at 55 years (Fig. 4,F,G). Dark material showed positive immunostaining for all sarcomeric proteins (desmin, myotilin and alpha-B crystallin) (Fig. 4,H,I) and presented a mild congophilia without apple-green birefringence under polarized light (Fig. 4,L,M). IHC for myosins revealed a large predominance of fibers expressing slow myosin in PII.1 with few immature small fibers expressing neonatal myosin. Ultrastructural study in PII.1 confirmed the presence of small nemaline bodies and revealed large areas of sarcomeric disorganization devoid of mitochondria with abundant electrodense material consistent with unstructured cores, probably corresponding to the dark areas devoid of enzymatic activity at optic microscopy (Fig. 4,N,O)

\subsection{Molecular data}

In order to identify the molecular cause of this peculiar core-rod myopathy, we performed a target NGS sequencing in the proband. The customized NGS panel for congenital myopathy allowed to identify a novel heterozygous missense mutation c.148G $>A$ p.(Gly50Ser) in exon 3 of ACTAl gene, segregating in all affected members in the family, consistent with a dominant inheritance. This variant is predicted to be pathogenic by Polyphen-2 and SIFT software and Gly50 is a highly conserved amino acid into the subdomain 2 of the $\alpha$-actin. Because of the peculiar clinical phenotype (myopathic face with SCM atrophy) and pseudomyotonic discharges at EMG study we also ruled out triplet and tetraplet expansions in DMPK and ZNF9 genes causing Myotonic Dystrophy type 1 and 2 respectively. No variants in other genes causing nemaline myopathies or non-dystrophic myotonias were detected. 


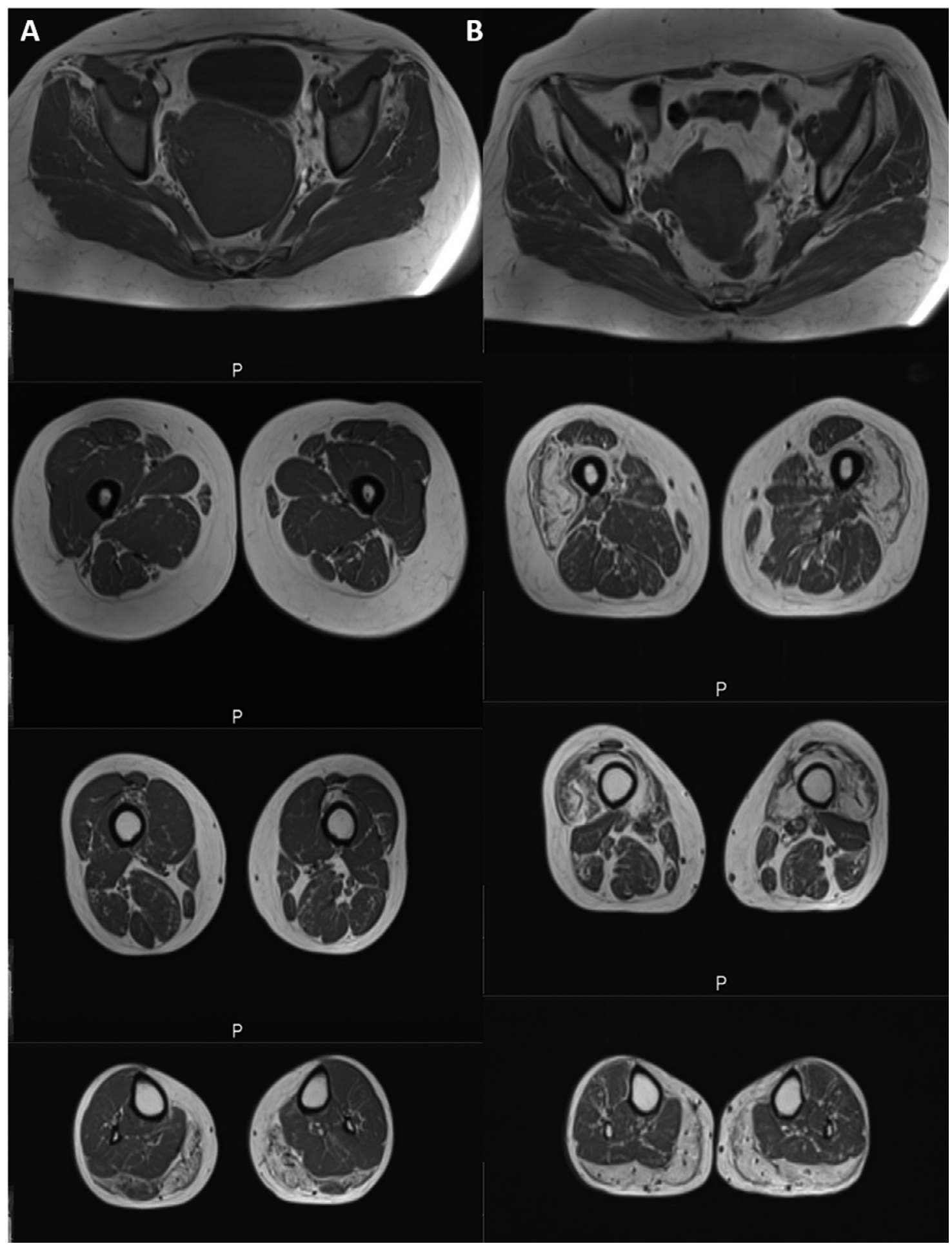

Fig. 3. Muscle MRI.

Similar pattern of fibro-fatty replacement in PII.1 (A) and PII.4 (B): rectus abdominis, gluteus minimus, vastus intermedius and gastrocnemii. This pattern was more evident in the older sister PII.4 (B) with more prominent quadriceps involvement. Posterior muscles of the thighs were also mildly affected.

\subsection{Functional assays of fibre contractility}

To study whether sarcomeric changes contribute to muscle weakness, we isolated permeabilized single muscle fibers from the biopsy of the patient. These fibers were exposed to incremental $\mathrm{Ca}^{2+}$ concentrations and the resulting forces were recorded. Note that of the 39 fibers isolated, 34 were type I fibers based on myosin heavy chain isoform composition (in accordance to type I fibers predominance in the biopsy). Thus, because of the low number of type II fibers, for the 

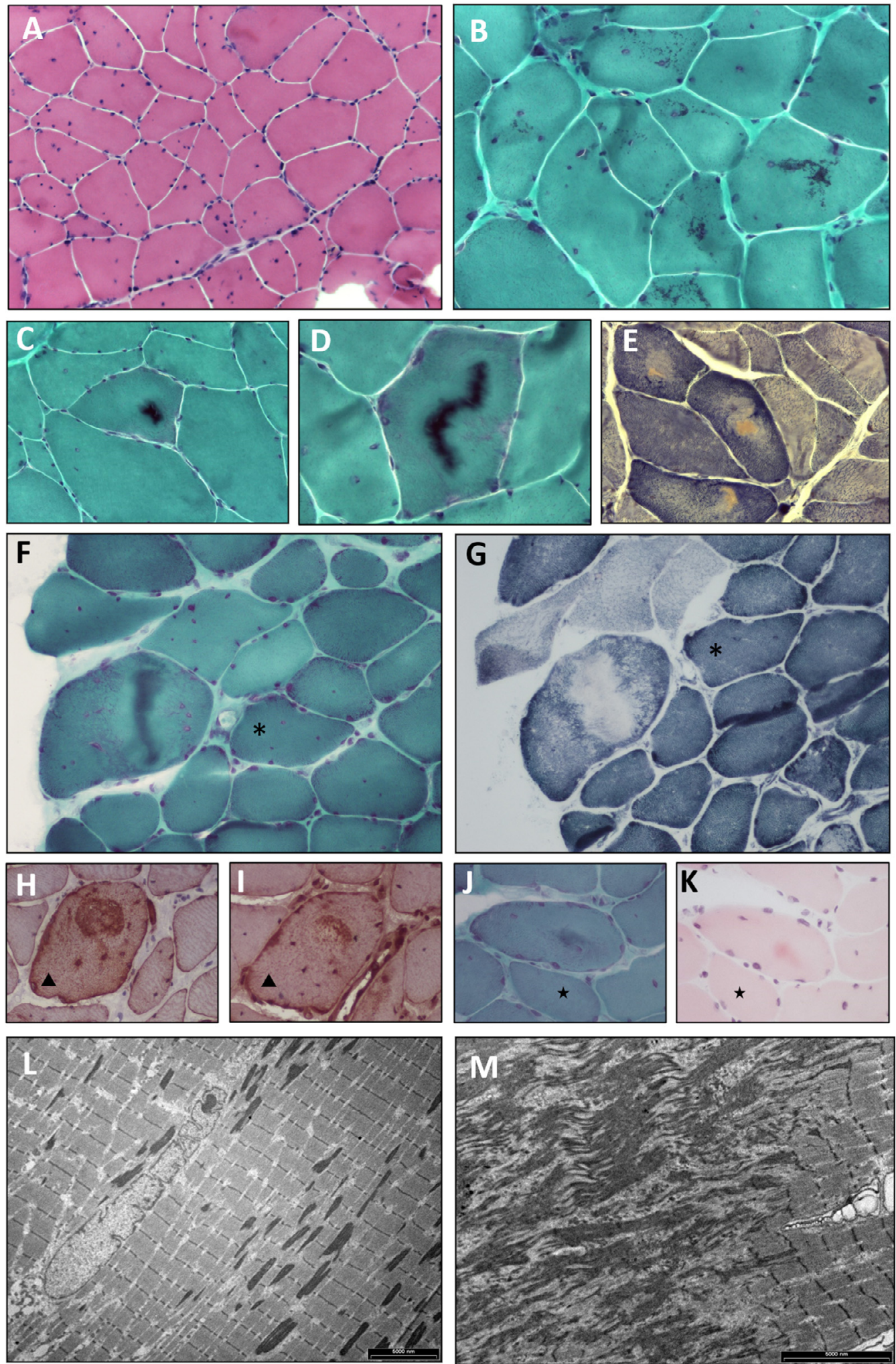

Fig. 4. Muscle biopsy.

A-E: proband's muscle biopsy (PII.4: biceps brachii and vastus lateralis, 39 ys). F-I: older sister's muscle biopsy (PII.1, vastus lateralis, 55ys). Prominent nuclear internalization (A), clusters of cytoplasmic and subsarcolemmal nemaline bodies (B) and cytoplasmic areas of dark material deposition with perilesional halo at GT stain (C,D,F,J), corresponding to irregular areas devoid of enzymatic activity at oxidative stains (E). Similar histopathological findings in the muscle biopsy of the older sister (F,G). Positive immunostaining for desmin (H) myotilin (I) of dark material with congophilia (K). Electron microscopy confirmed the presence of small nemaline rods (L) and revealed large areas of sarcomeric disorganization with abundant smeared electrodense material (M). [Serial sections: F-G, H-I, J-K. Stains, magnifications: HE,10x (A); GT, 20x (B,C,F), 40x (D,L); NADH, 20x (E,G); Myotilin, 40x (H); Desmin, 40x (I); Congo Red, 40x (M)]. 

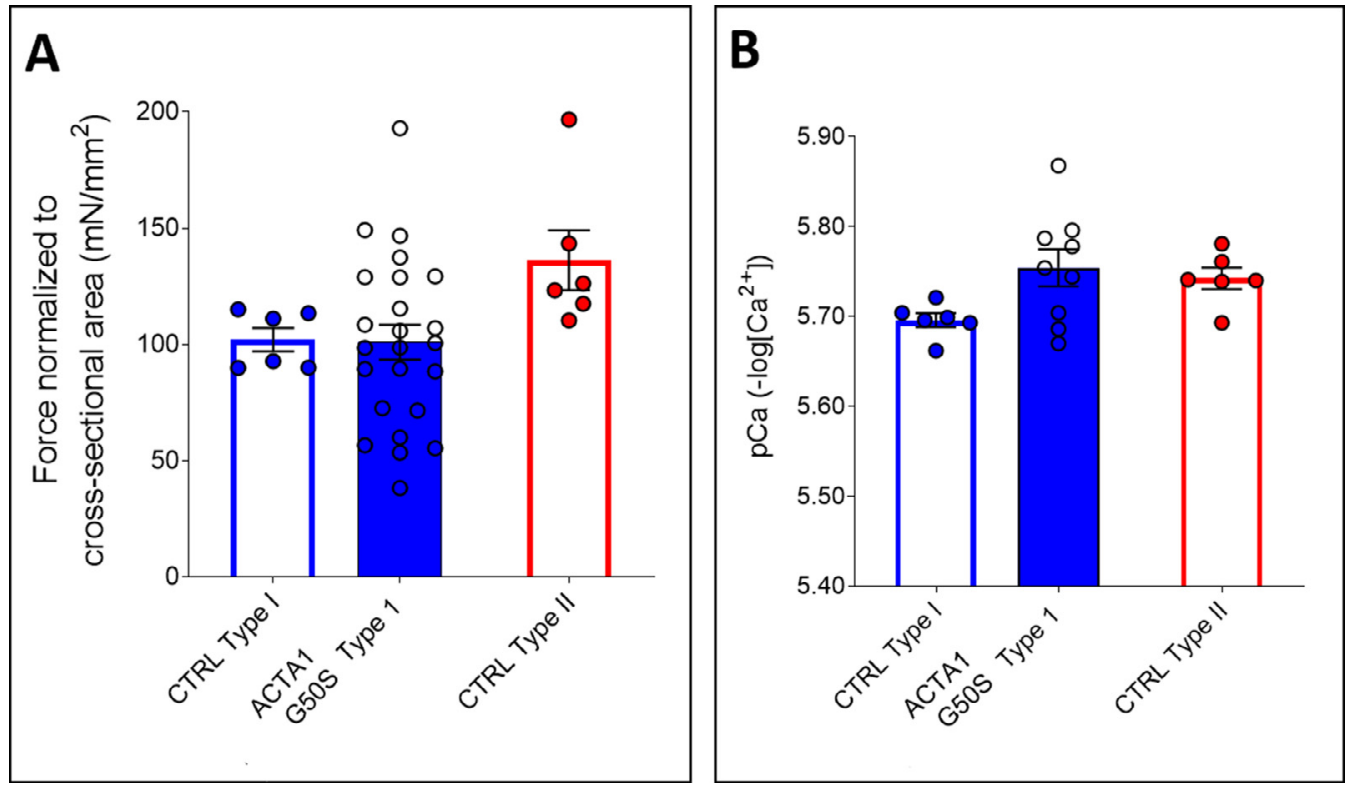

Fig. 5. Functional assays of fiber contractility.

Maximal active tension in type1 fibers from PII.1 muscle biopsy. Maximal active tension of type 1 muscle fiber from PII.4 (left panel, filled blue column) was comparable to type I fibers from control subjects (empty blue column) (A). Higher $\mathrm{pCa}_{50}$ in the PII.1 type1 fibers (right panel, filled blue column) compared to the type I fibers of the control subjects (empty blue column) (B). [Each dot in the patient bar reflects the result of one myofiber; each dot in the control bar reflects the average of one patient (with the average based on 8-15 myofibers)].

patient we only show data from the type I fibers. Maximal active force (at pCa 4.5) was normalized to the cross-sectional area of the fiber (i.e. tension). In our patient, maximal active tension was comparable to the tension of type I fibers from control subjects (Fig 5,A) but the $\mathrm{pCa}_{50}$, a measure of the calcium sensitivity of force, appears slightly higher in the patient's type I fibers compared to those of control subjects (Fig 5,B).

\section{Discussion}

Late, adult-manifesting congenital myopathy represents the milder spectrum of ACTAl myopathies and the minority of ACTAl cases reported [8,15]. Most of these cases had mild facial involvement with proximal weakness of upper limbs and variable proximo-distal weakness in lower limbs, most frequently reported as facioscapuloperoneal presentation [3,30,30,44,45]. Accordingly, the few reports including a muscle MRI study showed diffuse fibro-fatty replacement in lower limbs predominant in the anterolateral compartment of the lower legs associated to posterior compartment of the thighs, sartorius and gluteus maximus $[3,46]$. Conversely, our patients shared a different pattern of muscle involvement characterized by gluteus minimus, rectus abdominis, quadriceps and gastrocnemii as the most affected muscles of the lower limbs. Moreover, clinical features presented some peculiarities: first, none of the affected subjects in our family presented distal lower limb weakness, due to prevalent involvement of gastrocnemii and possible compensation by soleus muscle; second, all patients presented predominant involvement of head-neck involvement with mild facial weakness, dysmorphic features and SMC atrophy, as frequently observed in myotonic dystrophy [47]. Histopathological study revealed unique findings. Besides slow fiber predominance and nemaline bodies, frequently observed in ACTA1 myopathies, we observed in both muscle samples the presence of unusual cores characterized by of dark material with peripheral halo at GT stain,. Similar histopathological lesions had been described in another family harbouring a different mutation c. $(757 \mathrm{G}>C)$ in ACTAl manifesting as distal nemaline myopathy [44]. Cores with similar dark appearance have also been reported in $R Y R I$ recessive myopathies (dusty cores) [48] and other conditions like neurogenic disorders can mimic core-like lesions with central dark area and peripheral halo (target fibers) [49]. RYR1 mutations can also lead to core-rod myopathy, as well as mutations in NEB, CFL2, and KBTBD13 genes [50]. While core-rod myopathy most frequently manifests as early onset severe congenital myopathy, late-adult onset core-rod myopathy has been less frequently observed in association to certain RYR1 and KBTBD13 mutations [51-53].

On the other hand, two different missense mutations affecting the Gly50 residue have been reported with different or uncertain phenotypes: 1) the p.(Gly50Asp), recently described in association with actinopathy with rimmed vacuoles and prominent finger flexor weakness [33] and 2) the p.(Gly50Cys), reported on the Leiden Open Variation Database without confirming its pathogenicity (https:// databases.lovd.nl/ shared/ genes/ACTA1). The high variability in clinico-pathological spectrum of ACTAl myopathies seems 
to be due, at least in part, to different mutations in ACTAl gene, because of similar phenotype among subjects of the same family. Nevertheless, different age at onset or clinical severity in the affected members of the same family suggest that other genetics or environmental factors would contribute to the individual phenotype. To date, more than 200 pathogenic variants in the ACTAl gene have been reported. As few polymorphic amino acid variants have been identified among hundreds of normal ACTAl alleles, it seems that most actin residues are critical for its function, in accordance with its high evolutionary conservation [54]. Mechanisms by which mutations in ACTAl contribute to contractile weakness depends on the type of mutation and include reduction in length of the thin filament and structural damage to myofibrils [10]. Knowledge on sarcomere contractility in NEM3 patients could have therapeutic implications [11]. The novel p.(Gly50Ser) variant in ACTA1 reported in our family, predicted to be deleterious by in silico analysis, affects a highly conserved Gly50 residue into the subdomain 2 of the $\alpha$-actin. This domain, together with subdomain 1 , constitutes a small domain of the helical filament of the protein where all monomers are oriented similarly, with both subdomains 1 and 2 located on the outer edge of the filament axis [54]. Subdomain 2 rotation seems to occur within F-actin following ATP hydrolysis and phosphate release. Evidences support an interaction between subdomain 2 , the nucleotide pocket and the C-terminus, suggesting that intranuclear rod formation could be functionally linked to nucleotide binding [54]. Similarly we speculate that Gly50ser mutation could lend binding properties for other sarcomeric proteins to subdomain 2 , possibly resulting in unusual cores formation, as detected by positive immunostaining for sarcomeric proteins in muscle biopsy.

Functional study in muscle fibers of our patient, surprisingly do not show alterations in type 1 fiber contractility as observed in the majority of ACTAl patients and other nemaline myopathies, but just a higher calcium sensitivity force observed only in the minority of ACTAl patients $[10,11,55]$. The findings suggest no major changes in thin filament length, but perhaps structural changes that enhance the binding of myosin.

In conclusion our findings expand the clinico-pathological spectrum of ACTA1-related myopathies and the genetic spectrum of core-rod myopathies.

\section{Disclosures}

All authors report no relevant disclosures and conflict of interest for this study.

All data of this study are available from the corresponding author, upon motivated request.

This study complies all the ethical and local standards.

\section{Funding}

This study did not receive any funding.

\section{References}

[1] Goebel HH, Laing NG. Actinopathies and myosinopathies. Brain Pathol 2009;19:516-22. https://doi.org/10.1111/j.1750-3639.2009.00287.x.

[2] Nowak KJ, Ravenscroft G, Laing NG. Skeletal muscle $\alpha$-actin diseases (actinopathies): pathology and mechanisms. Acta Neuropathol 2013;125:19-32. https://doi.org/10.1007/s00401-012-1019-z.

[3] Hernandez-Lain A, Cantero D, Camacho-Salas A, Toldos O, Esteban I, Pascual I, et al. Autosomal dominant distal myopathy with nemaline rods due to p.Glu197Asp mutation in ACTA1. Neuromuscul Disord 2019;29:247-50. https://doi.org/10.1016/j.nmd.2018.12.001.

[4] Hutchinson DO, Charlton A, Laing NG, Ilkovski B, North KN. Autosomal dominant nemaline myopathy with intranuclear rods due to mutation of the skeletal muscle ACTA1 gene: clinical and pathological variability within a kindred. Neuromuscul Disord 2006;16:113-21. https: //doi.org/10.1016/j.nmd.2005.11.004.

[5] Ilkovski B, Nowak KJ, Domazetovska A, Maxwell AL, Clement S, Davies KE, et al. Evidence for a dominant-negative effect in ACTA1 nemaline myopathy caused by abnormal folding, aggregation and altered polymerization of mutant actin isoforms. Hum Mol Genet 2004;13:1727-43. https://doi.org/10.1093/hmg/ddh185.

[6] Nowak KJ, Wattanasirichaigoon D, Goebel HH, Wilce M, Pelin K, Donner $\mathrm{K}$, et al. Mutations in the skeletal muscle alpha-actin gene in patients with actin myopathy and nemaline myopathy. Nat Genet 1999;23:208-12. https://doi.org/10.1038/13837.

[7] O'Grady GL, Best HA, Oates EC, Kaur S, Charlton A, Brammah S, et al. Recessive ACTA1 variant causes congenital muscular dystrophy with rigid spine. Eur J Hum Genet 2015;23:883-6. https://doi.org/10. 1038/ejhg.2014.169.

[8] Agrawal PB, Strickland CD, Midgett C, Morales A, Newburger DE, Poulos MA, et al. Heterogeneity of nemaline myopathy cases with skeletal muscle alpha-actin gene mutations. Ann Neurol 2004;56:8696. https://doi.org/10.1002/ana.20157.

[9] Nowak KJ, Sewry CA, Navarro C, Squier W, Reina C, Ricoy JR, et al. Nemaline myopathy caused by absence of alpha-skeletal muscle actin. Ann Neurol 2007;61:175-84. https://doi.org/10.1002/ana.21035.

[10] Winter JM de, Joureau B, Lee E-J, Kiss B, Yuen M, Gupta VA, et al. Mutation-specific effects on thin filament length in thin filament myopathy. Ann Neurol 2016;79:959-69. https://doi.org/10.1002/ana. 24654.

[11] Joureau B, de Winter JM, Conijn S, Bogaards SJP, Kovacevic I, Kalganov A, et al. Dysfunctional sarcomere contractility contributes to muscle weakness in ACTA1-related nemaline myopathy (NEM3). Ann Neurol 2018;83:269-82. https://doi.org/10.1002/ana.25144.

[12] Levesque L, Del Bigio MR, Krawitz S, Mhanni AA. A de novo dominant mutation in ACTA1 causing congenital nemaline myopathy associated with a milder phenotype: expanding the spectrum of dominant ACTA1 mutations. Neuromuscul Disord 2013;23:239-42. https://doi.org/10.1016/j.nmd.2012.12.004.

[13] Laing NG, Dye DE, Wallgren-Pettersson C, Richard G, Monnier N, Lillis $\mathrm{S}$, et al. Mutations and polymorphisms of the skeletal muscle alpha-actin gene (ACTA1). Hum Mutat 2009;30:1267-77. https://doi. org/10.1002/humu.21059.

[14] Ilkovski B, Cooper ST, Nowak K, Ryan MM, Yang N, Schnell C, et al. Nemaline myopathy caused by mutations in the muscle alpha-skeletalactin gene. Am J Hum Genet 2001;68:1333-43. https://doi.org/10.1086/ 320605

[15] Moreno C de AM, Abath Neto O, Donkervoort S, Hu Y, Reed UC, Oliveira ASB, et al. Clinical and histologic findings in ACTA1-related nemaline myopathy: case series and review of the literature. Pediatr Neurol 2017;75:11-16. https://doi.org/10.1016/j.pediatrneurol.2017.04. 002.

[16] Domazetovska A, Ilkovski B, Kumar V, Valova VA, Vandebrouck A, Hutchinson DO, et al. Intranuclear rod myopathy: molecular pathogenesis and mechanisms of weakness. Ann Neurol 2007;62:597608. https://doi.org/10.1002/ana.21200.

[17] Ravenscroft G, Wilmshurst JM, Pillay K, Sivadorai P, Wallefeld W, Nowak KJ, et al. A novel ACTA1 mutation resulting in a severe 
congenital myopathy with nemaline bodies, intranuclear rods and type I fibre predominance. Neuromuscul Disord 2011;21:31-6. https://doi.org/ 10.1016/j.nmd.2010.08.005.

[18] Schröder JM, Durling H, Laing N. Actin myopathy with nemaline bodies, intranuclear rods, and a heterozygous mutation in ACTA1 (Asp154Asn). Acta Neuropathol 2004;108:250-6. https://doi.org/10. 1007/s00401-004-0888-1.

[19] Goebel HH, Anderson JR, Hübner C, Oexle K, Warlo I. Congenital myopathy with excess of thin myofilaments. Neuromuscul Disord 1997;7:160-8.

[20] Kaindl AM, Rüschendorf F, Krause S, Goebel H-H, Koehler K, Becker C, et al. Missense mutations of ACTA1 cause dominant congenital myopathy with cores. J Med Genet 2004;41:842-8. https: //doi.org/10.1136/jmg.2004.020271.

[21] Hung RM, Yoon G, Hawkins CE, Halliday W, Biggar D, Vajsar J. Cap myopathy caused by a mutation of the skeletal alpha-actin gene ACTA1. Neuromuscul Disord 2010;20:238-40. https://doi.org/10.1016/ j.nmd.2010.01.011.

[22] Laing NG, Clarke NF, Dye DE, Liyanage K, Walker KR, Kobayashi Y, et al. Actin mutations are one cause of congenital fibre type disproportion. Ann Neurol 2004;56:689-94. https://doi.org/10.1002/ana. 20260.

[23] Tadokoro K, Ohta Y, Sasaki R, Takahashi Y, Sato K, Shang J, et al. Congenital myopathy with fiber-type disproportion accompanied by dilated cardiomyopathy in a patient with a novel p.G48A ACTA1 mutation. J Neurol Sci 2018;393:142-4. https://doi.org/10.1016/j.jns. 2018.08.015.

[24] Clarke NF, Ilkovski B, Cooper S, Valova VA, Robinson PJ, Nonaka I, et al. The pathogenesis of ACTA1-related congenital fiber type disproportion. Ann Neurol 2007;61:552-61. https://doi.org/10.1002/ana. 21112.

[25] Sewry CA, Holton JL, Dick DJ, Muntoni F, Hanna MG. Zebra body myopathy is caused by a mutation in the skeletal muscle actin gene (ACTA1). Neuromuscul Disord 2015;25:388-91. https://doi.org/ 10.1016/j.nmd.2015.02.003.

[26] Wallgren-Pettersson C, Laing NG. Report of the 83rd ENMC International Workshop: 4th Workshop on Nemaline Myopathy, 22-24 September 2000, Naarden, The Netherlands. Neuromuscul Disord 2001;11:589-95.

[27] Colombo I, Scoto M, Manzur AY, Robb SA, Maggi L, Gowda V, et al. Congenital myopathies: natural history of a large pediatric cohort. Neurology 2015;84:28-35. https://doi.org/10.1212/WNL. 0000000000001110.

[28] Selcen D. Severe congenital actin related myopathy with myofibrillar myopathy features. Neuromuscul Disord 2015;25:488-92. https://doi. org/10.1016/j.nmd.2015.04.002.

[29] Stenzel W, Prokop S, Kress W, Huppmann S, Loui A, Sarioglu NME, et al. Fetal akinesia caused by a novel actin filament aggregate myopathy skeletal muscle actin gene (ACTA1) mutation. Neuromuscul Disord 2010;20:531-3. https://doi.org/10.1016/j.nmd.2010.06.008.

[30] Kao JC, Liewluck T, Milone M. A novel ACTA1 mutation causing progressive facioscapuloperoneal myopathy in an adult. J Clin Neurosci 2018;53:261-2. https://doi.org/10.1016/j.jocn.2018.04.044.

[31] Jungbluth H, Sewry CA, Brown SC, Nowak KJ, Laing NG, Wallgren-Pettersson C, et al. Mild phenotype of nemaline myopathy with sleep hypoventilation due to a mutation in the skeletal muscle alpha-actin (ACTA1) gene. Neuromuscul Disord 2001;11:35-40.

[32] Jain RK, Jayawant S, Squier W, Muntoni F, Sewry CA, Manzur A, et al. Nemaline myopathy with stiffness and hypertonia associated with an ACTA1 mutation. Neurology 2012;78:1100-3. https://doi.org/10.1212/ WNL.0b013e31824e8ebe.

[33] Liewluck T, Niu Z, Moore SA, Alsharabati M, Milone M. ACTA1myopathy with prominent finger flexor weakness and rimmed vacuoles. Neuromuscul Disord 2019;29:388-91. https://doi.org/10.1016/j.nmd. 2019.02.012.

[34] Donkervoort S, Chan SHS, Hayes LH, Bradley N, Nguyen D, Leach ME, et al. Cytoplasmic body pathology in severe ACTA1-related myopathy in the absence of typical nemaline rods. Neuromuscul Disord 2017;27:531-6. https://doi.org/10.1016/j.nmd.2017.02.012.

[35] Garibaldi M, Fionda L, Vanoli F, Leonardi L, Loreti S, Bucci E, et al. Muscle involvement in myasthenia gravis: expanding the clinical spectrum of myasthenia-myositis association from a large cohort of patients. Autoimmun Rev 2020:102498. https://doi.org/10.1016/j.autrev. 2020.102498.

[36] Hollingsworth KG, de Sousa PL, Straub V, Carlier PG. Towards harmonization of protocols for MRI outcome measures in skeletal muscle studies: consensus recommendations from two TREAT-NMD NMR workshops, 2 May 2010, Stockholm, Sweden, 1-2 October 2009, Paris, France. Neuromuscul Disord 2012;22(Suppl 2):S54-67. https: //doi.org/10.1016/j.nmd.2012.06.005.

[37] Garibaldi M, Tasca G, Diaz-Manera J, Ottaviani P, Laschena F, Pantoli D, et al. Muscle MRI in neutral lipid storage disease (NLSD). J Neurol 2017;264:1334-42. https://doi.org/10.1007/s00415-017-8498-8.

[38] Fischer D, Kley RA, Strach K, Meyer C, Sommer T, Eger K, et al. Distinct muscle imaging patterns in myofibrillar myopathies. Neurology 2008;71:758-65. https://doi.org/10.1212/01.wnl.0000324927.28817.9b.

[39] Ottenheijm CAC, Buck D, de Winter JM, Ferrara C, Piroddi N, Tesi C, et al. Deleting exon 55 from the nebulin gene induces severe muscle weakness in a mouse model for nemaline myopathy. Brain 2013;136:1718-31. https://doi.org/10.1093/brain/awt113.

[40] Hill AV. The possible effects of the aggregation of the molecules of haemoglobin on its dissociation curves. J Physiol 1910;40:iv-vii.

[41] Walker JS, Li X, Buttrick PM. Analysing force-pCa curves. J. Muscle Res. Cell. Motil. 2010;31:59-69.

[42] Joureau B, de Winter JM, Conijn S, Bogaards SJP, Kovacevic I, Kalganov A, et al. Dysfunctional sarcomere contractility contributes to muscle weakness in ACTA1-related nemaline myopathy (NEM3). Ann. Neurol. 2018;83:269-82.

[43] Ottenheijm CAC, Witt CC, Stienen GJ, Labeit S, Beggs AH, Granzier H. Thin filament length dysregulation contributes to muscle weakness in nemaline myopathy patients with nebulin deficiency. Hum. Mol. Genet. 2009;18:2359-69.

[44] Liewluck T, Sorenson EJ, Walkiewicz MA, Rumilla KM, Milone M. Autosomal dominant distal myopathy due to a novel ACTA1 mutation. Neuromuscul Disord 2017;27:742-6. https://doi.org/10.1016/ j.nmd.2017.05.003.

[45] Zukosky K, Meilleur K, Traynor BJ, Dastgir J, Medne L, Devoto M, et al. Association of a novel ACTA1 mutation with a dominant progressive scapuloperoneal myopathy in an extended family. JAMA Neurol 2015;72:689-98. https://doi.org/10.1001/jamaneurol.2015.37.

[46] Castiglioni C, Cassandrini D, Fattori F, Bellacchio E, D'Amico A, Alvarez $\mathrm{K}$, et al. Muscle magnetic resonance imaging and histopathology in ACTA1-related congenital nemaline myopathy. Muscle Nerve 2014;50:1011-16. https://doi.org/10.1002/mus.24353.

[47] Ashizawa T, Sarkar PS. Myotonic dystrophy types 1 and 2. Handb Clin Neurol 2011;101:193-237. https://doi.org/10.1016/ B978-0-08-045031-5.00015-3

[48] Garibaldi M, Rendu J, Brocard J, Lacene E, Fauré J, Brochier G, et al. "Dusty core disease" (DuCD): expanding morphological spectrum of RYR1 recessive myopathies. Acta Neuropathol Commun 2019;7:3. https://doi.org/10.1186/s40478-018-0655-5.

[49] von Fellenberg A, Lin S, Burgunder J-M. Disturbed trafficking of dystrophin and associated proteins in targetoid phenomena after chronic muscle denervation. Neuropathol Appl Neurobiol 2004;30:255-66. https://doi.org/10.1046/j.0305-1846.2004.00529.x.

[50] Jungbluth H, Treves S, Zorzato F, Sarkozy A, Ochala J, Sewry C, et al. Congenital myopathies: disorders of excitation-contraction coupling and muscle contraction. Nature Reviews Neurology 2018;14:151-67. https: //doi.org/10.1038/nrneurol.2017.191.

[51] Garibaldi M, Fattori F, Bortolotti CA, Brochier G, Labasse C, Verardo $\mathrm{M}$, et al. Core-rod myopathy due to a novel mutation in BTB/POZ domain of KBTBD13 manifesting as late onset LGMD. Acta Neuropathol Commun 2018;6:94. https://doi.org/10.1186/ s40478-018-0595-0. 
[52] Monnier N, Romero NB, Lerale J, Nivoche Y, Qi D, MacLennan DH, et al. An autosomal dominant congenital myopathy with cores and rods is associated with a neomutation in the RYR1 gene encoding the skeletal muscle ryanodine receptor. Hum Mol Genet 2000;9:2599-608. https: //doi.org/10.1093/hmg/9.18.2599.

[53] Sambuughin N, Yau KS, Olivé M, Duff RM, Bayarsaikhan M, Lu S, et al. Dominant mutations in KBTBD13, a member of the BTB/Kelch family, cause nemaline myopathy with cores. The American Journal of Human Genetics 2010;87:842-7. https://doi.org/10.1016/j.ajhg.2010.10. 020 .
[54] Sparrow JC, Nowak KJ, Durling HJ, Beggs AH, Wallgren-Pettersson C, Romero N, et al. Muscle disease caused by mutations in the skeletal muscle alpha-actin gene (ACTA1). Neuromuscul Disord 2003;13:51931. https://doi.org/10.1016/s0960-8966(03)00101-9.

[55] de Winter JM, Ottenheijm CAC. Sarcomere dysfunction in nemaline myopathy. J Neuromuscul Dis 2017;4:99-113. https://doi.org/10.3233/ JND- 160200. 